\title{
Cars gone wild: the major contributor to unintended acceleration in automobiles is pedal error
}

\author{
Richard A. Schmidt ${ }^{1,2 *}$ and Douglas E. Young ${ }^{3,4}$ \\ Department of Psychology, University of California, Los Angeles, Los Angeles, CA, USA \\ 2 Human Performance Research, Marina del Rey, CA, USA \\ ${ }^{3}$ Exponent Inc., Failure Analysis Associates, Los Angeles, CA, USA \\ ${ }^{4}$ Department of Kinesiology, California State University, Long Beach, CA, USA
}

\section{Edited by:}

Gabriele Wulf, University of Nevada,

Las Vegas, USA

\section{Reviewed by:}

Gabriele Wulf, University of Nevada,

Las Vegas, USA

Rebecca Lewthwaite, Rancho Los

Amigos National Rehabilitation Center

and the University of Southern

California, USA

*Correspondence:

Richard A. Schmidt, Human

Performance Research, 135 Lighthouse

Mall, Marina del Rey, CA 90292-5992,

USA.

e-mail: raschmidt@hp-research.com

\begin{abstract}
"Unintended-acceleration" automobile accidents typically begin when the driver first enters the car, starts the engine, and intends to press his/her right foot on the brake while shifting from Park to a drive gear (Drive or Reverse). The driver reports an unintended (uncommanded) full-throttle acceleration, coupled with a loss of braking, until the episode ends in a crash. Pedal misapplications - where the right foot contacts the accelerator instead of the brake that was intended - have been linked to these accidents (Schmidt, 1989, 1993) which, in the 1980s, were thought to occur only at the start of a driving cycle (and/or with the car in Park). But, in 1997, we identified over 200 pedal errors as the cause of accidents reported in the North Carolina database; these crashes occurred during the driving cycle (Schmidt et al., 1997), and/or with the vehicle in a gear other than Park. Our present work provides a more thorough analysis of these North Carolina Police Accident Reports from 1979 to 1995. The vast majority of pedal misapplications (over $92 \%$ ) (a) occurred during the driving cycle, (b) were generally in "unhurried" conditions, and (c) were categorically separate from those events referred to as unintended-acceleration episodes at start-up. These ideas are explanatory for the recent (2009-2010) surge of unintendedacceleration reports, perhaps even suggesting that all of these crashes are caused by pedal errors, and that none of them are based on some vehicle defect(s).
\end{abstract}

Keywords: automobile accidents, unintended-acceleration, pedal misapplications, vehicle defect

\section{INTRODUCTION}

The term "unintended acceleration" (UA - sometimes called "sudden acceleration") was coined in the 1980s to describe a type of automobile accident that was attracting considerable attention at the time. In these episodes, the driver would report that, as he/ she was initiating a driving cycle, after starting the engine, placing the right foot lightly on the brake (to prevent the car from "creeping"), and shifting from Park to a drive gear (usually Park or Reverse, depending on the situation), the vehicle would go to an uncommanded (i.e., unintended) wide-open-throttle condition, coupled with an apparent loss of braking effectiveness (Pollard and Sussman, 1989; Reinhart, 1989; Schmidt, 1989, 1993). The episode would often end with a crash. In the 1980s, there was a surge of UA reports, representing almost every brand of automobile. This situation resulted in a frantic search by three federal government agencies, various auto makers, and several private research firms, for some electrical/mechanical defect that would cause these events. None was ever found. This naturally resulted in considerable publicity (e.g., newspaper and magazine articles, and even a "60 Minutes" television report; CBS, 1986) and substantial public awareness.

Over the next few decades, this situation gradually faded from public awareness. Recently, however, in 2009-2010, a similar situation has seemingly returned. For many of us who worked on this problem in the 1980s, the current situation brings the problem again to mind again, creating the suspicion that the same phenomena are still operating (or are operating again).
In the 1980s, a number of researchers began to suspect that these episodes might have a human-error component (e.g., Schmidt, 1989; Reinhart, 1994), and that a search for an electro-mechanical defect might be misguided. The essential idea was that the driver, intending to put the right foot on the brake, would occasionally place the foot on the accelerator by mistake. Then, when the car moved when a drive gear was engaged, the driver would press harder on the "brake" to stop it, the vehicle would go even faster, resulting in more "brake" application, usw., until the vehicle soon was in a wide-open-throttle condition; the driver (who was invariably panicked) was rendered incapable of diagnosing the situation in the few seconds before a crash occurred.

In the 1980s, these accidents were mainly associated with the start of a driving cycle (and/or otherwise initiating driving with the vehicle in Park gear, such as at a fast-food drive-through or at an outside bank-teller's station). These types of accidents led to National Highway Traffic Safety Administration's (NHTSA) formal definition which tended to confine UA crashes to purely start-up events (Pollard and Sussman, 1989).

Less well known are the frequency and type of pedal errors that occur during the driving cycle (i.e., with the vehicle already in Drive or Reverse). These events do not fall easily under the above NHTSA definition, even though (subsequent to the error) they seem to have many features in common with UA (full throttle, relatively long duration: several seconds or longer). In 1997, we (Schmidt et al., 1997) found a surprising number and variety of pedal errors leading to accidents, but which were not the "official," NHTSA-defined UA situation (because they did not start with the vehicle in Park or at low initial speed). 
In our initial work (Schmidt et al., 1997), we examined the North Carolina Police Accident Report database for the years 1979 and 1980 to determine the kinds of pedal errors that were made, the contributing factors, and the frequency of these events. We discovered 219 accidents for which the driver admitted having made a pedal error as a direct cause of the crash. These pedal errors occurred in many different driving situations (i.e., all with the vehicle being in one of the drive gears other than Park when the episode began). We were surprised that these accidents appeared to represent a fundamentally different type of accident initiation from that previously classified (by NHTSA; Pollard and Sussman, 1989) as UA. One principal difference is that the drivers in our pedal-error investigation knew (and admitted) that they had made an error, and offered this information to the investigating authorities. However, the drivers in NHTSA-defined UA episodes typically did not know that they had made a pedal error; in fact, the latter drivers were typically emphatic that they did not make a pedal error.

This distinction means that, if a pedal error caused an NHTSAdefined UA episode, the driver would report the cause in terms such as accelerator malfunction, stuck throttle, failed brakes, etc. However, for the second class of pedal-error accident (caused by an admitted pedal error, at speed, and with the vehicle not being in Park), a different type of accident description would be given by the driver; here language such as hit (i.e., the wrong pedal), miss (the brake), slipped (from brake to accelerator), usw. In the present paper, we expanded our search for this second type of pedal-misapplication crash, using North Carolina data from the years 1979 through 1995. Here, we report our examination of these two classes of accidents, and their relative frequencies, asking whether they can be considered as separate phenomena or as variants of the same phenomenon.

\section{MATERIALS AND METHODS}

The accident-report database from North Carolina contains over one million accidents of almost all types, where the accident was sufficiently serious to warrant a police report. The database includes the verbatim, written statement of the investigating officer describing how the accident happened, based in part on statements made by the driver(s) of the involved vehicle(s) and/or eye-witnesses or passengers. These narratives can be searched for various key words, depending on the overall research goal. Our keyword evaluation of these narrative statements produced two different searches: one for recognized and/or admitted pedal errors, and a second for NHTSAdefined UA episodes where the driver stated that his/her right foot was on the brake, and was not on the accelerator (i.e., pedal error was not admitted). A key question was whether or not these two searches would result in overlapping subsets of the accident database.

We first searched for keywords such as brake, foot, gas, pedal, and acc (for accelerate, accelerator, etc.), resulting in more than 94,000 accident reports. Next, we searched this subset with more detailed keywords to isolate accidents thought to be caused by pedal misapplications, such as miss, slip, instead, etc. This trimmed the set to about 20,000 accidents for which we were at least suspicious that a pedal error had led to the crash.

The next step was to read the remaining narratives to verify that the accident was, in fact, related to a pedal error. This process eliminated many cases which were not associated with pedal misapplications (e.g., "The driver swerved to miss a dog"). The final result was a set of 3740 accidents that satisfied our pedal-error criteria.
Such a subset will surely underestimate the total number of such accidents, as many drivers are reluctant to admit their error; and, some crashes are very minor and do not result in accident reports. And, of course, an unknown number of pedal errors may have been corrected before a crash actually occurred.

In order to find NHTSA-defined UA events, we reviewed the pedal-error accidents identified in this later study to determine if any of these cases could also be construed as an NHTSA-defined "unintended-acceleration" event, where a driver (a) shifts from Park to either Drive or Reverse at the start of a driving cycle, (b) incurs a sudden and unexpected vehicle acceleration, (c) reports a perceived failure of the brakes, and (d) reports a malfunction of the throttle or accelerator pedal. This analysis, however, produced no crashes that fit the NHTSA criteria for UA.

We then searched the accident database (for the years from 1979 to 1995) using key words believed to characterize NHTSA-defined UA episodes based on the accident narratives. These words included sudden, malfunction, runaway, stuck, shift, throttle, gear, control (for out of control), and produced over 43,000 accident records. We then reduced the set by combining various key words (e.g., accelerator stuck), and adding other keywords, which included terms such as park, jump, start, unexpect (for unexpected or unexpectedly), etc., to eliminate those accidents that were clearly not pedal-error based (e.g., accidents involving a car door stuck open). We then read and categorized these narratives.

\section{RESULTS}

Of the accidents identified, two distinct sets of pedal errors were found: (a) 3740 accidents which were clearly caused by pedal misapplication, and (b) a non-overlapping 39 reports that were NHTSAdefined UA episodes. This represents an important finding, in that our earlier understanding of pedal errors was that they were associated mainly with the start of the driving cycle. With this evidence, it is now clear that pedal misapplications can occur frequently in several additional ways, perhaps as Rogers and Wierwille (1988) have found in simulators.

\section{CLASSIFICATIONS}

The first of several classifications concerned whether the driver was parking or driving. "Parking" involved those situations where the car was in a parking lot, home garage, or was being parked in a parking space on the street. "Driving" included all other situations, where it was clear that the vehicle was being driven on the road or highway (and therefore was not in Park). Some of the crashes that occurred while driving might well have been classed as parking accidents, however - e.g., where the vehicle was being driven to a parking space.

Next, for each of these accidents we coded the pedal misapplication as either a slip from the brake, or as hitting the "wrong pedal" (the accelerator) without first slipping from the brake. Also, we classified the scenario as (1) hurried, (2) unhurried, or (3) distracted to identify the urgency or other extenuating circumstances involved in the attempted brake application.

For the driving accidents, we made one additional classification concerning the circumstances of the pedal application. These were classified as accidents in which the vehicle or driver was (1) turning, (2) slowing ("normally"), (3) stopped, or (4) other. For the parking accidents, we classified them as occurring in the forward or backward direction. 


\section{PARKING ACCIDENTS}

Only 279 of the 3740 accidents were involved in what we considered parking operations (see Table 1). These were divided between moving forward (216) and backward (63). In terms of mechanisms of the misapplication, 126 accidents were caused by slips from the brake, and 153 occurred because the driver hit the "wrong pedal" for some reason. Only 19 of the accidents were classified as hurried, with 213 not hurried, and 47 distracted. It is curious that the nearly half of the accidents occurring in parking operations happened because the driver for some reason hit the "wrong pedal," with over three-quarters of the accidents being under nonhurried conditions.

\section{DRIVING ACCIDENTS}

The overwhelming majority of accidents caused by pedal misapplications occurred under driving conditions ( $92.5 \%$, see Table 2). Of these, 2640 were caused by a slip, whereas the remainder (821) resulted from the driver pressing the "wrong pedal." Also, there were more unhurried driving accidents (2938) as compared to those hurried (268) or distracted (255). This trend is similar to that seen in the parking actions.

The circumstances surrounding the pedal misapplications were then separated into three categories (see Table 2). The largest single category was slowing ("normally"), with 793 accidents, followed by stopped and turning, with 673 and 231 accidents, respectively. We could not classify 1641 accidents from the narratives alone.

We found an apparent interaction between the type of pedal misapplication and the driving circumstances surrounding it (see Table 3). First, for accidents that occurred while the vehicle was stopped, most (604) were associated with slipping of the foot from the brake, whereas only 69 were caused by hitting the "wrong pedal."

Table 1 | Parking accidents (279/3740 or $7.5 \%)$.

\begin{tabular}{lc}
\hline Characteristic & Number \\
\hline Slip & 126 \\
"Wrong pedal" & 153 \\
Forward direction & 216 \\
Backward direction & 63 \\
Hurried & 19 \\
Unhurried & 213 \\
Distracted & 47 \\
\hline
\end{tabular}

Table 2 | Driving accidents (3461/3740 or 92.5\%).

\begin{tabular}{lc}
\hline Characteristic & Number \\
\hline Slip & 2640 \\
"Wrong pedal" & 821 \\
Hurried & 268 \\
Unhurried & 2938 \\
Distraction & 255 \\
Slowing ("normally") & 793 \\
Turning & 231 \\
Stopped & 673 \\
Other & 1641
\end{tabular}

On the other hand, for accidents wherein the driver was making a turn, most (134) were associated with the driver hitting the "wrong pedal," whereas only 97 of these turning accidents involved slips from the brake.

\section{UNINTENDED-ACCELERATION EPISODES}

Keyword-search results for NHTSA-defined UA episodes produced only 39 accidents (Table 4). In these accidents, the drivers' accident description to the reporting officer indicated that the vehicle suddenly accelerated immediately or soon after the vehicle was placed into Drive or Reverse from either Park or Neutral. In these accidents, there was a fairly uniform description of the accident by the drivers. Nearly all of the accidents occurred in a parking lot, driveway, or parking space; one incident took place at an outdoor service window of a bank. Seventy-two percent (28/39) of the accidents involved a forward acceleration of the vehicle. And, in the majority of these accidents $(62 \%, 24 / 39)$, the driver indicated that the accelerator (or throttle) malfunctioned (e.g., hung, stuck); none of the drivers attributed their accidents to human error.

\section{DISCUSSION}

Consistent with our earlier findings (Schmidt et al., 1997), these data clearly contradict earlier ideas that pedal misapplications are associated only with the start of a driving cycle, such as in the NHTSA-defined UA episodes (e.g., Pollard and Sussman, 1989; Schmidt, 1989). We find that the overwhelming majority of the pedal misapplications (about 99\%) occurred after initial start-up, and a surprisingly small percentage (only about $1 \%$ ) were NHTSAdefined UA episodes.

The findings also provide interesting insight about how such simple limb movements in automobiles lead to on-road accidents of various types. Contrary to the notion that pedal errors occurred mainly as a result of being hurried (or distracted) - both of which could lead to a fast response, and, therefore, a less-accurate response (Schmidt et al., 1979) - a majority of the pedal misapplications

Table 3 | Pedal-error type as a function of driving circumstances.

\begin{tabular}{lcc}
\hline Action & \multicolumn{2}{c}{ Type of pedal error } \\
\cline { 2 - 3 } & Slip & "Wrong pedal” \\
\hline Stopped & 604 & 69 \\
Slowing & 613 & 180 \\
Turning & 97 & 134
\end{tabular}

Table 4 | NHTSA-defined unintended-acceleration events.

\begin{tabular}{lc}
\hline Driving circumstances & Number \\
\hline Parking & 38 \\
Other & 1 \\
Forward direction & 28 \\
Backward direction & 11 \\
Accelerator problem & 24 \\
Problem unknown & 15 \\
Human error & 0
\end{tabular}


occurred under unhurried conditions. Also, the type of error where the driver hit the "wrong pedal" occurs more frequently in driving situations that involve turning, perhaps indicating that there is an influence of the upper limb or body that creates a more erroneous lower-limb condition. Clearly, these findings on pedal misapplications point out the need to understand more completely the processes that produce pedal-based accidents on the road.

Several aspects of our data deserve further study. Two main classes of explanations have been proposed for pedal errors: errors in response choice (e.g., turn right rather than left), and errors in response execution (e.g., moving slightly too far). Schmidt (1989) has argued that UAs behave like response-execution errors in many ways, sensitive to the same factors that influence variability in human aiming movements, and are unlikely to be recognized as errors by drivers who have just made them. Many of the pedal errors that occur during driving, however, and which are reported as such by drivers, may be of this type (e.g., Rabbitt and Rodgers, 1977; Reason, 1990); such errors are susceptible to being recognized by performance-monitoring mechanisms.

Our data here can help us understand some fundamental principles of pedal misapplications. The NHTSA-defined UA events appear to be caused chiefly by an error in aiming the foot toward the brake during a nearly simultaneous shift from Park to one of the drive gears, Drive or Reverse (Schmidt, 1989). This type of error was recognized in the 1980s, and interlocks were designed to prevent them. This interlock prevented this type of UA by locking the shift lever in Park until the brake pedal was depressed. If a pedal misapplication should be made at the start of a driving cycle (i.e., pressing the accelerator rather than the brake that was intended), the vehicle will not move, as it is safely locked in Park. These interlocks were overwhelmingly successful in reducing NHTSA-defined UAs (Reinhart, 1994), decreasing their incidence by approximately $60 \%$. Also this success of the shift interlocks served as a crucial test of the hypotheses that UA was caused by (a) pedal misapplications

\section{REFERENCES}

CBS. (1986). 60 Minutes. New York: Columbia Broadcasting System, November 25, 1986.

Pollard, J., and Sussman, E. D. (1989). An Examination of Unintended Acceleration. Washington, DC: US Department of Transportation (DOTHS-8-7-367).

Rabbitt, P., and Rodgers, B. (1977). What does man do after he makes an error? An analysis of response programming. Q. J. Exp. Psychol. 29, 727-743.

Reason, J. (1990). Human Error. Cambridge: Cambridge University Press.

Reinhart, W. (1989). Alleged Sudden Unwanted Acceleration. Washington, DC: US Department of Transportation (ODI Case No. C86-01).
Reinhart, W. (1994). The Effect of Countermeasures to Reduce the Incidence of Sudden Acceleration Accidents. Washington, DC: US Department of Transportation (NHTSA), 821-845 (Paper No. 94 S5 O 07).

Rogers, S. B., and Wierwille, W.W. (1988). The occurrence of accelerator and brake pedal actuation errors during simulated driving. Hum. Factors 30, 71-81.

Schmidt, R. A. (1989). Unintended acceleration: a review of humanfactors contributions. Hum. Factors 31, 345-364.

Schmidt, R.A. (1993). "Unintended acceleration: human performance considerations," in Automotive Ergonomics, eds B. Peacock and W. Karwowski (London, England: Taylor and Francis), 431-451.

vs. (b) some electro-mechanical vehicle defect. Indeed, if NHTSAdefined UA is caused by a vehicle defect, then locking the transmission in park until one of the driver's feet is on the brake, preventing a UA event, supports a pedal-misapplication theory, and rejects a theory involving some vehicle defect.

To this understanding, we now add the finding that these pedal misapplications can occur in other ways than at vehicle start-up. They can occur during a driving cycle when the vehicle has already been shifted from Park to Drive or Reverse, under which conditions the shift interlocks can no longer be effective (because the vehicle is no longer in Park). And, this latter type of UA (after vehicle start-up) appears to be far more frequent than the episodes occurring at start-up; our data show that only about $1 \%$ of the UA episodes are of the type defined by NHTSA at start-up, the remainder being caused by pedal misapplications after start-up. We think that his last observation helps us understand why all of the UA episodes were not eliminated by the car-makers' adoption of some variant of the shift interlocks in the 1980s; some of the pedal misapplications probably occurred after the car had been shifted from Park.

Finally, in the situation involving UA in the 1980s, despite formal searches by a number of research groups, no electro-mechanical defect was ever found that would account for the accidents. And, since we have considerable evidence that UAs can be caused by pedal misapplications, we are left with the possibility that all of the 1980s UAs were pedal-error based. With the present-day surge in reported UA events, the situation is almost the same. Granted, some have suggested that the UAs can be caused by sticking pedals or by floor mats trapping the accelerator pedal; but these explanations can account for only a small number of these events. This raises the possibility that, as with UA in the 1980s, the majority, or even essentially all of the present-day UAs may be based on pedal misapplications, with none being caused by some vehicle defect(s) (Schmidt, 2010).

Schmidt, R.A. (2010). Breaking Bad. New York: New York Times, Opinion and Editorial Pages, March 11, 2010.

Schmidt, R. A., Young, D. E., Ayres, T. J., and Wong, J. R. (1997). "Pedal misapplications: Their frequency and variety revealed through police accident reports," in Proceedings of the Human Factors Society 41st Annual Meeting (Santa Monica, CA: Human Factors and Ergonomics Society), 1023-1027.

Schmidt, R. A., Zelaznik, H. N., Hawkins, B., Frank, J. S., and Quinn, J. T. (1979) Motor-output variability: a theory for the accuracy of rapid motor acts. Psychol. Rev. 86, 415-451.

Conflict of Interest Statement: The authors declare that the research was conducted in the absence of any commercial or financial relationships that could be construed as a potential conflict of interest.

Received: 02 November 2010; accepted: 03 November 2010; published online: 25 November 2010.

Citation: Schmidt RA and Young DE (2010) Cars gone wild: the major contributor to unintended acceleration in automobiles is pedal error. Front. Psychology 1:209. doi: 10.3389/fpsyg.2010.00209

This article was submitted to Frontiers in Movement Science and Sport Psychology, a specialty of Frontiers in Psychology. Copyright () 2010 Schmidt and Young. This is an open-access article subject to an exclusive license agreement between the authors and the Frontiers Research Foundation, which permits unrestricted use, distribution, and reproduction in any medium, provided the original authors and source are credited. 